\title{
Optimization of Laser Parameters and Dimple Geometry Using PCA-Coupled GRG
}

\author{
Saravanan Kandasamy Ganesan ${ }^{1}$ - Thanigaivelan Rajasekaran2,* \\ ${ }^{1}$ Sona College of Technology, India \\ 2 Muthayammal Engineering College, India
}

Stainless steel (SS316L) is applied in numerous fields due to its intrinsic properties. In this study, micro-dimples were fabricated on SS316L. The effects of laser process parameters, such as frequency, average power, and pulse duration, on the average dimple diameter, dimple distance, and dimple depth were studied using an L $_{9}$ orthogonal array. The analysis of variance (ANOVA) and multi-objective optimization technique, principal-component-analysis-coupled grey relational grade (GRG), was used to optimize laser process parameters on output responses. The optimal machining parameter settings obtained for the highest GRG peak value of 0.2642 are $15 \mathrm{kHz}$ (frequency), $12 \mathrm{~W}$ (average power), and $1500 \mathrm{~ns}$ (pulse duration). The ANOVA results showed that average power is the most influential factor, contributing 86.40 $\%$ to performance measures (average dimple diameter $(\varphi)$, dimple distance (d), and depth (I). Moreover, the effect of process parameters was studied using mean effect plots, and the micro-dimple quality was analysed using SEM micrographs.

Keywords: laser, micro-dimple, principal component analysis, grey relational grade, stainless steel

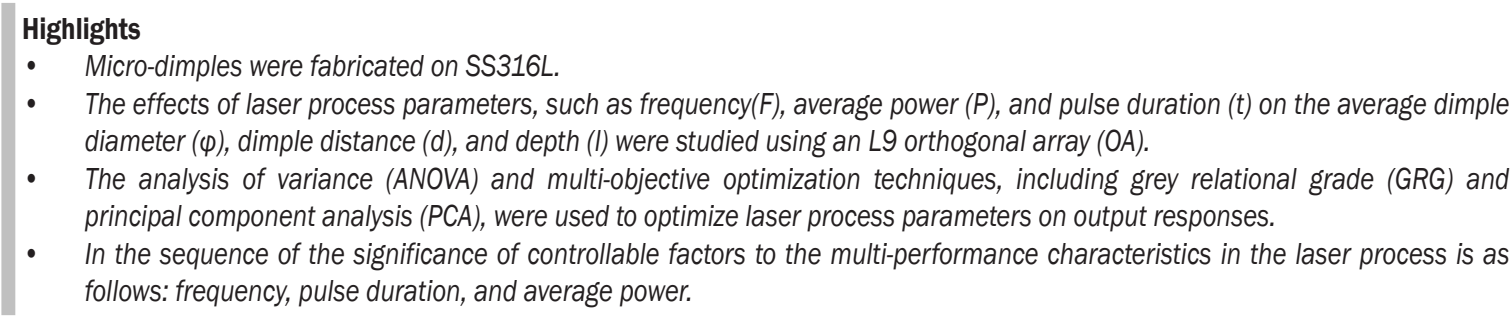

\section{INTRODUCTION}

Stainless steel finds applications in food processing, pharmaceutical equipment, medical devices, potable water, and wastewater treatment industries due to its improved corrosive resistance property. Although stainless steel alloys have excellent properties, including corrosion resistance, higher strength and hardness, their tribological action is not satisfactory in biomedical applications, in particular for hip prostheses that require sliding contact [1]. Moreover, for biomedical applications, stainless steel alloy surfaces must be modified for tissue attachment and wear resistance [2]. Hence, their surface properties must be improved, and numerous methods, such as plasma and flame coatings, shot peening, thermal oxidation, nanocomposite coatings, and ball burnishing, are available [3]. Each process has advantages and limitations. In this study, laser texturing was employed to improve the wear resistance of the stainless steel alloy surfaces. Laser texturing can be used to modify the SS-316L surface by generating micro-dimples to improve its properties under service conditions. Kovac1 and Seçer [4] investigated the effects of different surface textures acquired through selective laser melting on the mechanical properties of AISI 316L stainless steel. Their results showed that the effects of surface textures and the area density on friction and wear properties were highly significant. Liew et al. [5] studied the effect of the dimple geometry with different textural shapes and orientations on friction and reported that geometric factors, such as the shape dimple area, area ratio, and dimple depth, considerably influence the load-carrying capacity of contacting surfaces. Yu et al. [6] investigated the effect of different dimple shapes on the tribological performance of surface textures. The dimple area ratio and depth considerably affect the friction reduction. According to the literature, surface modification plays a key role in improving the mechanical properties of materials. The fabrication of micro-dimples by using laser and control of process parameters on geometrical features were considered in [6]. Li et al. [7] fabricated the micro-dimples by using laser peen texturing on SUS304 stainless steel and found that the diameter, depth, and aspect ratio of the micro-dimples increased with laser power density. Dai et al. [8] used masked laser ablation to fabricate a micro-dimple array on a substrate surface with a pulsed Nd:YAG laser, investigated the influence of processing parameters on the micro- 
dimple morphology, and developed clean periphery micro-dimples. Ahmed et al. [9] reviewed the effect of the geometrical parameters of micro-dimples on piston/cylinder assembly and concluded that the high dimple depth is unfavourable for development for starved lubrication. The most crucial parameter to enhance the tribological performance of piston ring/ cylinder assembly is the aspect ratio [9]. Won and Kim [10] developed masked laser surface texturing to produce hundreds of micro-patterns. The number of laser pulses substantially affects uniform pattern formation [10]. Puoza et al. [11] micro-machined 45 steel, GCr15 steel, and grey cast iron materials by using laser processing technologies and analysed the features, including the diameter and depth, of the ablated micro-dimples. The diameter and depth of the micro-dimples generally increased with an increase in the laser fluence for all the materials [11]. Behera et al. [12] fabricated micro-channels and micro-dimples with different dimensions on anSS-304 alloy surface by using the pulsed Nd:YAG laser beam and studied the effects of process parameters, such as laser scanning speed, current, laser pulse frequency, and pulse duration, on the diameter and depth of microfeatures. The dimensions of micro-features decreased an increase in the scanning speed and increased with an increase in the pulse frequency, pulse duration, and current [12]. Researchers have synthesized various micro-features with diverse dimensions for numerous applications. To improve the adherence of coatings with substrates, Lamraoui et al. [13] fabricated microdimples with a diameters and depths of $60 \mu \mathrm{m}$ to 90 $\mu \mathrm{m}$ and $60 \mu \mathrm{m}$ to $120 \mu \mathrm{m}$, respectively. Teleginski et al. [14] textured micro-dimples of $200 \mu \mathrm{m}$ diameter on a material. Gajrani et al. [15] developed micro-dimples with the diameter and depth of $250 \mu \mathrm{m}$ and $50 \mu \mathrm{m}$ on a cutting tool for lubrication retention.

Researchers have used lasers to modify alloy surfaces for various applications, and the optimal use of laser process parameters for efficient production and high accuracy requires the proper control of input parameters. Although lasers have been used for alloy surface modification, no systematic study has reported the optimization of the laser process by using a multiobjective optimization technique. Hence, in this study, micro-dimples were fabricated on SS316L, and the effects of laser factors such as frequency $(F)$, average power $(P)$, and pulse duration $(t)$ on the average dimple diameter $(\varphi)$, dimple distance $(d)$, and depth $(l)$ were studied using an $\mathrm{L}_{9}$ orthogonal array (OA). The analysis of variance (ANOVA) and multi-objective optimization techniques, including grey relational grade (GRG) and principal component analysis (PCA), were used to optimize laser process parameters on output responses. Moreover, the laser parameters must be optimized on geometrical parameters; otherwise, the laser parameters may negatively affect output performance.

\section{METHODS}

In this study, the SS316L alloy was selected as the workpiece material. Surgical 316L stainless ssteel was purchased from SAIL and its composition (wt \%) are17.20 Cr, $12.60 \mathrm{Ni}, 2.40 \mathrm{Mo}, 1.95 \mathrm{Mn}, 1 \mathrm{Si}, 0.03$ $\mathrm{C}, 0.02 \mathrm{~N}$, and balance Fe [16]. Nine samples with the length, width, and thickness of $25 \mathrm{~mm}, 25 \mathrm{~mm}$, and $5 \mathrm{~mm}$, respectively, were used. The specimens were cleaned with acetone and immersed in an ultrasonic vibrator for $1 \mathrm{~min}$, and again, were cleaned with deionized water. The factors considered for the experiments are frequency $(F)$ in $\mathrm{kHz}$, average power $(P)$ in Watts, and pulse duration $(t)$ in nanoseconds. The factors are selected based on the preliminary experiments. Each factor has three levels, as seen in Table 1. OA was selected based on the degrees of freedom (DoF). DoF is used to determine how many runs are required to develop a parameter estimate. Each parameter estimate attracts one DoF from the total number of DoFs offered. The numbers of factors and levels were three and three, respectively. Thus, the multiplication of the number of factors with the difference between the number of levels and 1 provides DoF. The selected OA should be higher than DoF; therefore, based on the relevant calculation, $3(3-1)$ provides six DoFs, and selected OA is larger than 6 , that is, $\mathrm{L}_{9} \mathrm{OA}[3]$. The average dimple diameter $(\varphi)$, dimple distance $(d)$, and depth $(l)$ were measured three times.

\section{EXPERIMENTAL}

Laser dimples were generated on the specimen according to the $\mathrm{L}_{9} \mathrm{OA}$ experimental design (Table 1). A fibre laser (CK-FB(A); Meera Laser Solutions) emitting a Gaussian beam at $1030 \mathrm{~nm}$ with the maximum output power and frequency of $20 \mathrm{~W}$ and $25 \mathrm{kHz}$, respectively, was used. The standoff distance was maintained at $0.7 \mathrm{~mm}$. The quality of the laser beam highly depends on its polarization state. In practice, laser texturing is normally performed by the beam with circular and chaotic polarization for the fibre laser. The dimples are made with multiple pulses, and the laser jumps from dimple to dimple. The spot diameter of the laser beam on stainless steel was $150 \mu \mathrm{m}$. The dimple depth and diameter 
Table 1. $\angle 9 O A$ and output responses

\begin{tabular}{cccccccccc}
\hline $\begin{array}{c}\text { Sample } \\
\text { number }\end{array}$ & $F[\mathrm{kHz}]$ & $P[\mathrm{~W}]$ & $T[\mathrm{~ns}]$ & $\varphi[\mu \mathrm{m}]$ & $d[\mu \mathrm{m}]$ & $l[\mu \mathrm{m}]$ & \multicolumn{3}{c}{$S T D E V$} \\
\hline 1 & 10 & 12 & 500 & 166.3 & 274.3 & 16.3 & 0.141 & 0.681 & 0.153 \\
\hline 2 & 10 & 14 & 1000 & 161.2 & 265.4 & 15.4 & 0.833 & 0.200 & 0.200 \\
\hline 3 & 10 & 16 & 1500 & 168.5 & 272.5 & 16.2 & 1.258 & 0.839 & 0.306 \\
\hline 4 & 15 & 12 & 1500 & 173.9 & 250.2 & 17.3 & 0.709 & 0.404 & 0.100 \\
\hline 5 & 15 & 14 & 500 & 171.7 & 252.1 & 16.8 & 1.159 & 0.153 & 0.231 \\
\hline 6 & 15 & 16 & 1000 & 157.2 & 300.5 & 15.6 & 0.757 & 0.500 & 0.458 \\
\hline 7 & 20 & 12 & 1000 & 134.9 & 335.3 & 13.9 & 0.586 & 0.252 & 0.361 \\
\hline 8 & 20 & 14 & 1500 & 140.4 & 340.2 & 14.2 & 0.306 & 0.902 & 0.306 \\
\hline 9 & 20 & 16 & 500 & 138.0 & 366.6 & 13.6 & 0.529 & 0.808 & 0.458 \\
\hline
\end{tabular}

were characterized and analysed using a non-contact 3D surface profilometer (made by Taylor Hobson) and scanning electron microscopy. The time of laser hatching was recorded from the laser machine display. Fig. 1 illustrates the schematic of the micro-dimple geometry.

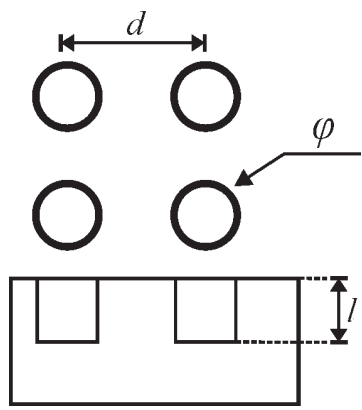

Fig. 1. Schematic of the micro-dimples on a substrate

\subsection{Grey Relational Analysis}

OA with the grey relational analysis (GRA) was used to determine the optimal machining parameters. In general, for GRG, each series is normalized by dividing the data into the original series by using their average [17]. Let the original reference and comparison sequences be $x_{i}^{*}(k)$ and $x_{i}(k)$, respectively, $i=1,2, \ldots$, $m ; k=1,2, \ldots, n$, where $m$ and $n$ are the total number of experiments and observed data, respectively. Data pre-processing is used to convert the original sequence into an identical sequence. Several data preprocessing methods can be used in GRA, depending on the characteristics of the original sequence.

If the target value of the original sequence is "thelarger-the-better", then the original sequence can be normalized (Eq. (1)) as follows:

$$
x_{i}^{*}(k)=\frac{x_{i}(k)-\min x_{i}(k)}{\max x_{i}(k)-\min x_{i}(k)} .
$$

If the purpose is "the-smaller-the-better", then the original sequence can be normalized (Eq.(2)) as follows:

$$
x_{i}^{*}(k)=\frac{\max x_{i}(k)-x_{i}(k)}{\max x_{i}(k)-\min x_{i}(k)} .
$$

After data pre-processing, a grey relational coefficient (GRC) can be calculated using the preprocessed sequences. GRC (Eq. (3)) are defined as follows:

$$
\xi_{i}(k)=\frac{\Delta_{\min }+\zeta \Delta_{\max }}{\Delta_{o i}(k)+\zeta \Delta_{\max }},
$$

where $\Delta_{o i}(k)$ is the deviation sequence of the reference sequence $x_{0}^{*}(k), x_{i}^{*}(k)$ is the comparability sequence, and $\zeta$ is the distinguishing coefficient, $\zeta$. GRG (Eq. (4)) is a weighted sum of the GRC defined as follows:

$$
\gamma_{i}=\frac{1}{n} \sum_{k=1}^{n} \xi_{i}(k)
$$

where GRG $\gamma_{i}$ represents the level of correlation between the reference and comparability sequences.

\subsection{Multivariate Statistical Technique}

PCA is a multivariate statistical technique developed by Pearson [18] and adapted by Hotelling [19]. In this technique, several correlated variables are transformed into fewer uncorrelated variables. These transformed uncorrelated variables are called principal components (PCs) and are the linear combinations of response variables. Following steps are involved in PCA [20]: 
Step 1. Formation of the decision matrix.

An array of original response variables is constructed as the decision matrix as in Eq. (5).

$$
\left[\begin{array}{ccccc}
\mathbf{x}_{11} & \mathbf{x}_{12} & \mathbf{x}_{13} & \ldots & \mathbf{x}_{1 n} \\
\mathbf{x}_{21} & \mathbf{x}_{22} & \mathbf{x}_{23} & \ldots & \mathbf{x}_{2 n} \\
\mathbf{x}_{31} & \mathbf{x}_{32} & \mathbf{x}_{33} & \ldots & \mathbf{x}_{3 n} \\
\vdots & \vdots & \vdots & \ddots & \vdots \\
\mathbf{x}_{m 1} & \mathbf{x}_{m 2} & \mathbf{x}_{m 3} & \ldots & \mathbf{x}_{m n}
\end{array}\right]
$$

where, $\mathbf{X}$ is the decision matrix of the response variables, $n$ and $m$ are the numbers of output characteristics and experimental observations, respectively. Output characteristics $n=3, m=$ 9, and decision matrix $\mathbf{X}$ is obtained as the GRC array of individual responses.

Step 2. The correlation coefficient array can be acquired from the decision matrix and is expressed as Eq.(6).

$$
C_{\alpha \beta}=\frac{\left(\operatorname{Cov}\left(x_{i}(\alpha)\right), x_{i}(\beta)\right)}{\sigma x_{i}(\alpha) \times \sigma x_{i}(\beta)},
$$

where, $\left(\operatorname{Cov}\left(x_{i}(\alpha), x_{i}(\beta)\right)\right.$ is the covariance of the sequences $\left(x_{i}(\alpha)\right),\left(x_{i}(\beta)\right), \sigma x_{i}(\alpha)$ is standard deviation (SD) $\sigma x_{i}(\alpha)$, and $\sigma x_{i}(\beta)=S D$ of $\sigma x_{i}(\beta)$. $\alpha=1,2, \ldots, n, \beta=1,2, \ldots, n . C_{\alpha \beta}$ elements are mainly the correlation coefficients of the entire combinations of performance measures such as dimple diameter, dimple distance, and dimple depth.

Step 3. Determination of Eigenvalues and vectors.

Eigenvalues and vectors are estimated from $C_{\alpha \beta}$ by using Eq. (7).

$$
E_{i k}=\left[C_{\alpha \beta}-\delta_{k} I_{m}\right],
$$

where, $\delta_{k}$ is the Eigenvalue such that $\sum_{k=1}^{n} \delta_{k}=n$, and $E_{i k}$ is the Eigenvector corresponding to Eigenvalue $\delta_{k}$.

Step 4. Estimation of PCs.

PCs are estimated using Eq. (8) and are arranged in the decreasing order of variations captured by them. Hence, the highest variability in the data is generally explained using the first principal component.

$$
P_{m k}=\sum_{i=1}^{n} x_{m}(i) \times E_{i k},
$$

where $P_{m k}$ is the first PC.
PCA GRG is estimated using Eq. (9).

$$
G_{i}=\in \sum_{i=1}^{n} W_{j}\left(\sum_{i=1}^{n} x_{m}(i)\right) .
$$

\section{RESULTS}

Performance measures, such as the average dimple diameter, dimple distance, and dimple depth, were considered. An increase in the average dimple diameter leads to the enhancement of lubrication storage for tribological applications and cell adherence in biomedical applications; thus, "the-larger-the-better" is considered the optimum condition. A decrease in the dimple depth and distance is effective to minimize the friction and wear; hence, "the-smaller-the-better" condition is used for the analysis. The original sequence is normalized using Eqs. (1) and (2) [21]. Subsequently, the deviation sequence is determined by subtracting the comparable sequence from the ideal sequence. GRC for output responses are calculated using Eq. (3) (Table 2). The PCA procedure is adapted to calculate the optimized weights. The GRC of the average dimple diameter, dimple distance, and depth are used as the elements of the decision matrix, which is used to calculate the correlation coefficient array and Eigenvalues using Eqs. (6) and (7), respectively. Table 3 presents the Eigenvalues and proportions for PCs. The first, second, and third PCs, that is, average dimple diameter, dimple distance, and dimple depth, respectively, account for $34.6 \%, 34.5 \%$, and $31 \%$ of variations, respectively, in GRG. Therefore, the accountability proportions of the first, second, and third PCs of $0.346,0.345$, and 0.310 are considered the optimum weights for the average dimple diameter, dimple distance, and dimple depth (Table 4).

Table 2. Calculated $\mathrm{GRC}$ value

\begin{tabular}{cccc}
\hline Sample number & $\varphi[\mu \mathrm{m}]$ & $d[\mu \mathrm{m}]$ & $l[\mu \mathrm{m}]$ \\
\hline 1 & 0.7196 & 0.7072 & 0.4066 \\
\hline 2 & 0.6056 & 0.7929 & 0.5068 \\
\hline 3 & 0.7831 & 0.7230 & 0.4157 \\
\hline 4 & 1.0000 & 1.0000 & 0.3333 \\
\hline 5 & 0.8986 & 0.9684 & 0.3663 \\
\hline 6 & 0.5387 & 0.5364 & 0.4805 \\
\hline 7 & 0.3333 & 0.4061 & 0.8605 \\
\hline 8 & 0.3679 & 0.3927 & 0.7551 \\
\hline 9 & 0.3520 & 0.3333 & 1.0000 \\
\hline
\end{tabular}

GRG was calculated by taking the average of GRCs matching to each process response by using 
Eq. (9). The overall estimation of multiple response process parameters was based on GRG (Table 5), and the experiment with the highest GRG is optimal among all the runs. The GRG for each $\mathrm{L}_{9} \mathrm{OA}$ experiment was used to optimize laser process parameters (Table 5). Subsequently, the GRG of each combination was ranked as per value, and a set of optimal machining parameter settings was obtained based on the highest GRG peak value of 0.2642 with $15 \mathrm{kHz}$ (frequency), $12 \mathrm{~W}$ (average power), and $1500 \mathrm{~ns}$ (pulse duration). The result indicated that experiment sample number 4 (Table 5) shows desirable responses among the nine experiments.

Table 3. Eigen values and proportions for PC

\begin{tabular}{lcc}
\hline PC & Eigen values & Proportion [\%] \\
\hline First & 0.1694 & 94.3 \\
\hline Second & 0.0077 & 4.3 \\
\hline Third & 0.0025 & 1.4 \\
\hline
\end{tabular}

Table 4. Accountability proportion of output responses

\begin{tabular}{lcccc}
\hline \multirow{2}{*}{$\begin{array}{l}\text { Output } \\
\text { parameters }\end{array}$} & \multicolumn{3}{c}{ Eigen vectors } & \multirow{2}{*}{ PC1 } \\
\cline { 2 - 4 } & PC2 & PC3 & \\
\hline$\varphi(\mu \mathrm{m})$ & 0.586 & 0.316 & -0.746 & 0.346 \\
\hline$d(\mu \mathrm{m})$ & 0.588 & 0.468 & 0.660 & 0.345 \\
\hline$l(\mu \mathrm{m})$ & -0.557 & 0.826 & -0.088 & 0.310 \\
\hline
\end{tabular}

Table 5. Ranking of parameter combination

\begin{tabular}{cccccc}
\hline $\begin{array}{c}\text { Sample } \\
\text { number }\end{array}$ & $F[\mathrm{kHz}]$ & $P[\mathrm{~W}]$ & $T[\mathrm{~ns}]$ & $G_{i}$ & Rank \\
\hline 1 & 10 & 12 & 500 & 0.2059 & 5 \\
\hline 2 & 10 & 14 & 1000 & 0.2131 & 4 \\
\hline 3 & 10 & 16 & 1500 & 0.2160 & 3 \\
\hline 4 & 15 & 12 & 1500 & 0.2642 & 1 \\
\hline 5 & 15 & 14 & 500 & 0.2524 & 2 \\
\hline 6 & 15 & 16 & 1000 & 0.1732 & 8 \\
\hline 7 & 20 & 12 & 1000 & 0.1739 & 7 \\
\hline 8 & 20 & 14 & 1500 & 0.1655 & 9 \\
\hline 9 & 20 & 16 & 500 & 0.1821 & 6 \\
\hline
\end{tabular}

Fig. 2 shows the micro-dimples machined for the optimum combination of parameters: $15 \mathrm{kHz}$ (frequency), $12 \mathrm{~W}$ (average power), and $1500 \mathrm{~ns}$ (pulse duration). The pile-up phenomenon around the dimples (Fig. 2a) contributes to limiting the contact surface, leading to decreased wear. Fig. $2 \mathrm{~b}$ shows the magnified view of micro-dimples and depicts the rough surface and re-solidified/re-cast areas. The re-solidified and re-cast layers on the micro- dimple resulted from the medium scanning speed, where material heat absorption decreased, leading to incomplete melting.
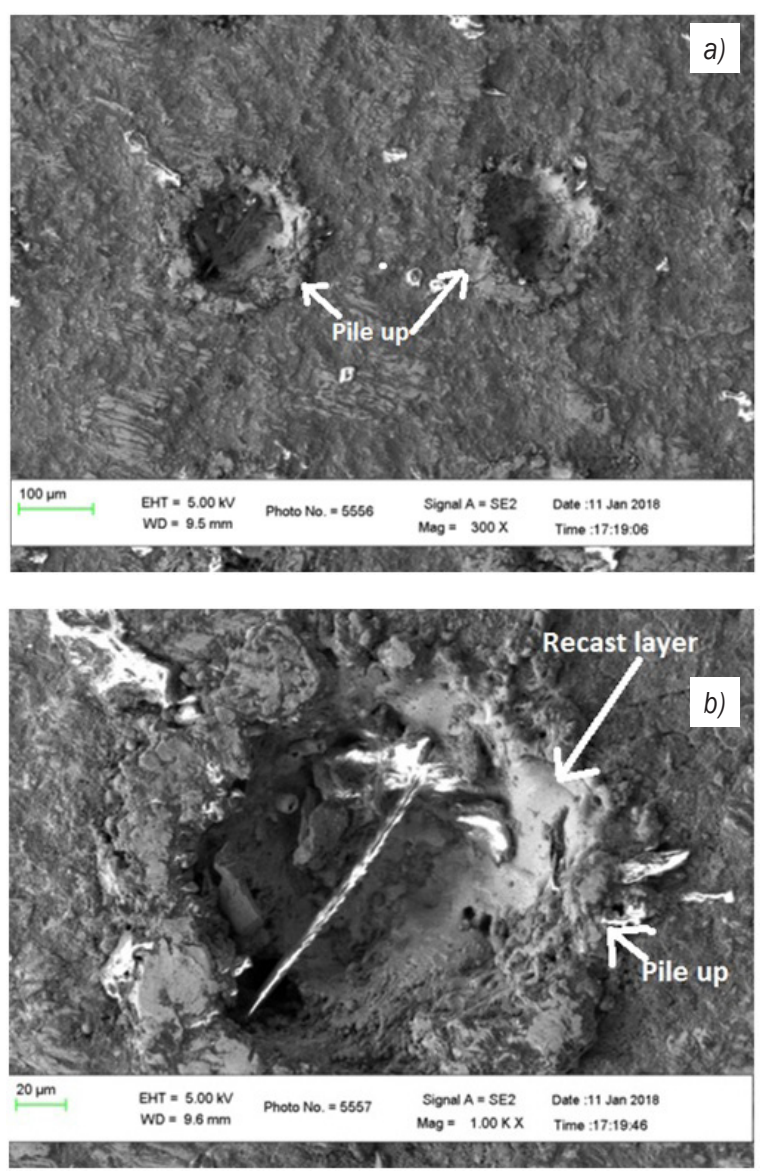

Fig. 2. Micro-dimple machined at optimum laser parameters, a) micro-dimple, b) a magnified view of the micro-dimple

The most significant factors of machining parameters are determined by estimating the difference between the maximum and minimum values of mean GRG.The values are 0.2299, 0.2147, and 0.2168 for the frequency, average power, and pulse duration, respectively. The result showed that the frequency $(0.2299)$ has the most decisive influence on multi-performance characteristics. In the sequence, the order of significance of controllable factors to multi-performance characteristics in the laser process is as follows: frequency, pulse duration, and average power. An increase in the pulse frequency improves laser spot extension leading to large and deep microdimples. At the frequency of $15 \mathrm{kHz}$, the pulsed laser beam exposed to the particular area of the workpiece causes heating and melting. With an increase in the pulse duration, the material exposure time increases, 
and hence, heat transfer to the workpiece increases. The pulse duration of $1500 \mathrm{~ns}$ contributes to the generation of wide and deep dimples.

\section{DISCUSSION}

Fig. 3 presents the mean effect plot for output performance. With the frequency increase, output performance increases. The pulse frequency is the number of pulses per unit of time. With the increase in the pulse frequency, the number of pulses per second also increases. When a pulsed laser beam focuses on the SS316L workpiece surface, the required area on the substrate heats, melts and/or evaporates. Moreover, overlapping between the laser spots results in material removal. Thus, an increase in the pulse frequency causes overlapping between the laser spots, resulting in highly prominent diameter micro-dimples. The further increase in the frequency and improper ejection of

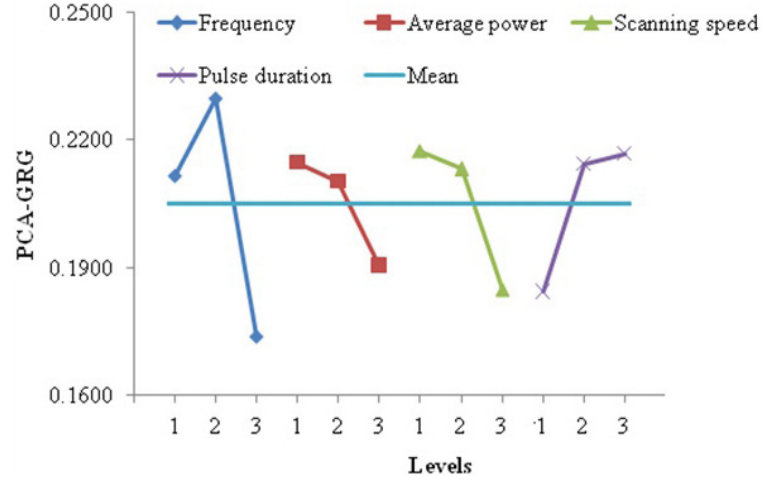

Fig. 3. Mean effect plot for output performance

molten materials hinder micro-dimple formation. The increase in laser power leads to a decrease in output performance. The increasing of power attributes to material removal, and the inadequate removal of molten materials reduces output performance. The
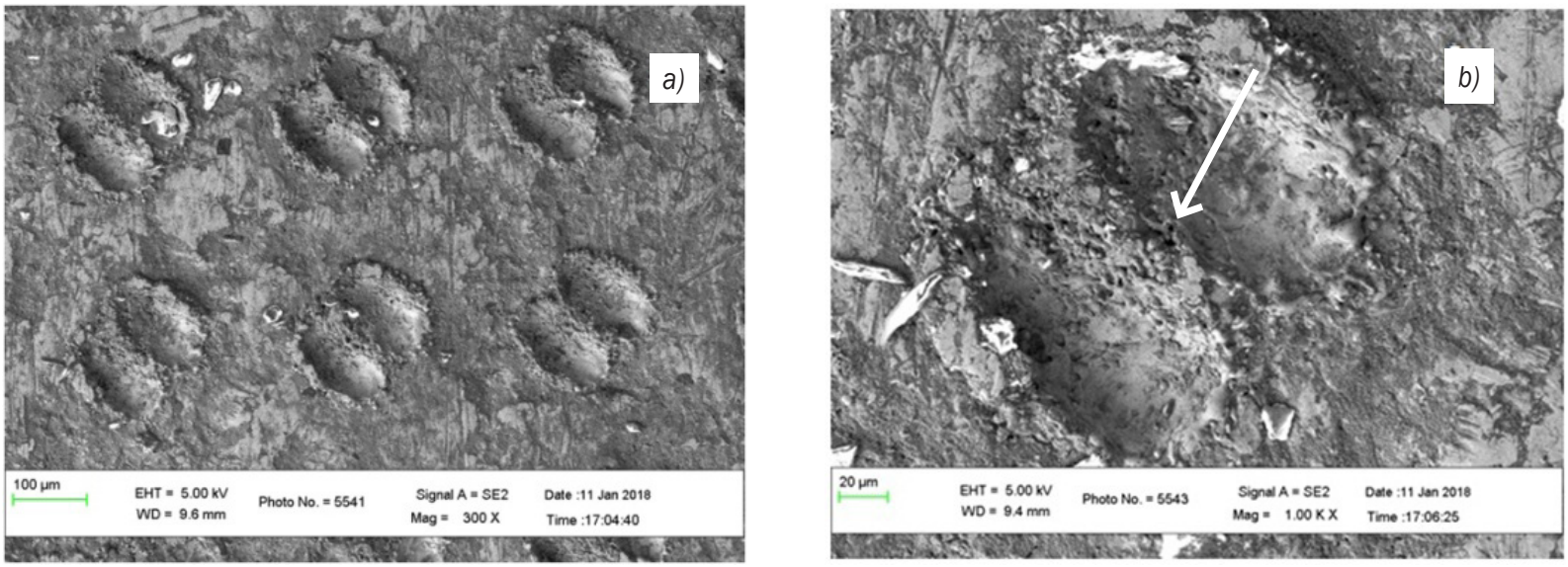

Fig. 4. a) Micro-dimples, and b) a magnified view of the dimple
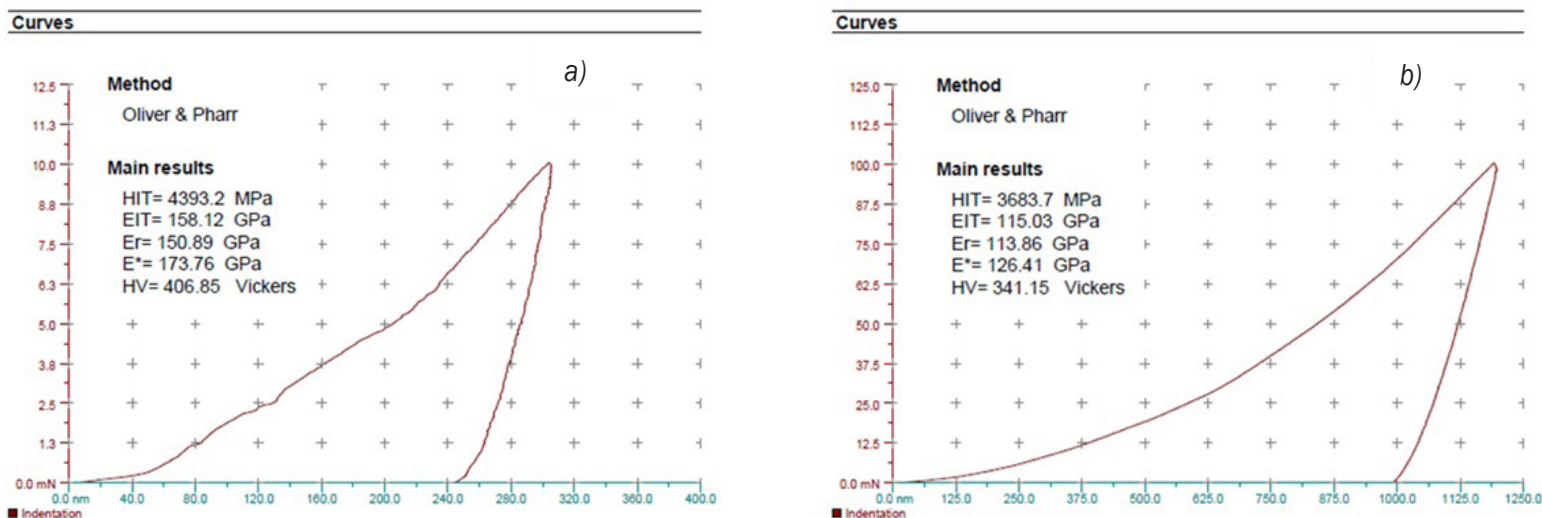

Fig. 5. Loading and unloading graphs of Vickers hardness for a) $10 \mathrm{mN}$, and b) $100 \mathrm{mN}$ 
important factor that significantly affects the microdimple quality is the interaction time between the laser beam and material. During interaction time, heat absorption of the material decreases, resulting in unfinished melting and the evaporation of the material surface, which leads to a decrease in the dimple diameter, distance, and depth. Fig. 4 illustrates the machined micro-dimples. The factor pulse duration exhibits an increasing trend in output responses. With an increase in the pulse duration, the laser exposure time on materials increases, contributing to an increase in output responses. Fig. 5 presents the loading and unloading graphs of Vickers hardness. The force was steadily lowered from $10 \mathrm{mN}$ to $0 \mathrm{mN}$ and 100 $\mathrm{mN}$ to $0 \mathrm{mN}$. The load and penetration depth of the indenter were continuously measured under the loadunload conditions. The nano-indentation test showed the hardness of $406.85 \mathrm{HV}$ and $341.15 \mathrm{HV}$ for 10 $\mathrm{mN}$ and $100 \mathrm{mN}$ loadings, respectively. The Vickers hardness of SS316L was $140 \mathrm{HV}$ in the non-laser irradiation area, and hardness increased almost three times. The arrow mark presented in Fig. 5 b shows the measurement positions on the dimple for the Vickers hardness test.

\subsection{ANOVA for PCA-GRG}

ANOVA is used to determine the most influencing parameter and to estimate the contribution percentage of laser process parameters [22] and [23]. ANOVA is a statistical tool employed to obtain the experimental results and to establish the performance of a group of analysed parameters. The total variance of an observed value can be calculated using the sum of squares (SS). Moreover, the sample variance is considered the mean square, which is obtained by dividing SS with the respective DoF. The $F$-value is the ratio of two variances and shows how far the values are scattered from the mean value. Larger $F$-values represent higher dispersion within the group. As per the ANOVA (Table 6), the average power is the most influential factor, contributing $86.40 \%$ to the performance measures, followed by the pulse duration [ns], and frequency [kHz], which contributes $11.66 \%$, and $1.77 \%$ to these measures. It is evident from the literature that the ablation mechanism is determined by applied power, and pulses have the least significance [24]. According to the comparison of the analytical mean results of PCA-GRG and ANOVA, the frequency and average power are the most significant parameters for dimple creation. This result variation was obtained because within the mean effect calculation, the difference between the two groups was considered, and in ANOVA, the variation between the sample means and the variation within each sample was considered.

\section{CONCLUSIONS}

In this study, micro-dimples were fabricated on SS316L, and the effects of laser process parameters such as the frequency, average power, and pulse duration on the average dimple diameter, dimple distance, and dimple depth were studied by using $\mathrm{L}_{9}$ $\mathrm{OA}$. The performance measures were optimized using PCA-coupled GRG. The following conclusions are drawn from the study.

1. The micro-dimples were successfully fabricated on the SS316L samples of $25 \mathrm{~mm} \times 25 \mathrm{~mm} \times 5$ $\mathrm{mm}$.

2. The optimal laser parameter setting for suitable micro-dimple generation for tribological applications is $15 \mathrm{kHz}$ (frequency), $12 \mathrm{~W}$ (average power), and $1500 \mathrm{~ns}$ (pulse duration).

3 . The most significant factors influencing microdimple generation are frequency and average power.

4. In the sequence, the order of significance of controllable factors to the multi-performance characteristics in the laser process is as follows: frequency, pulse duration, and average power.

5. With the increase in laser power, output performance decreases.

6. The development of a research model for predicting the number of micro-dimples for

Table 6. ANOVA results

\begin{tabular}{lccccc}
\hline Factors & DoF & SS & MS & F-value & Percentage contribution [\%] \\
\hline$F$ & 2 & 0.0000901 & 0.00004505 & 12.18 & 1.7786 \\
\hline$P$ & 2 & 0.0043769 & 0.00218845 & 591.47 & 86.4060 \\
\hline$t$ & 2 & 0.0005911 & 0.00029555 & 79.88 & 11.6691 \\
\hline Error & 2 & 0.0000074 & 0.0000037 & 1 & 0.1460 \\
\hline Total & 8 & 0.0067852 & 0.00084815 & 684.53 & $\sim 100$ \\
\hline
\end{tabular}


given area will be considered for further studies using the RSM model.

\section{ACKNOWLEDGEMENTS}

This manuscript was edited by editingindia.

\section{REFERENCES}

[1] Fellah, M., Labaïz, M., Assala, O., lost, A., Dekhil, L. (2013). Tribological behaviour of AISI 316L stainless steel for biomedical applications. Tribology - Materials, Surfaces \& Interfaces, vol. 7, no. 3, p. 135-149, D0l:10.1179/1751584X $13 \mathrm{Y} .0000000032$.

[2] Bekmurzayeva, A., Duncanson, W.Jr., Azevedo, H.S., Kanayeva, D. (2018). Surface modification of stainless steel for biomedical applications: Revisiting a century-old material. Materials Science and Engineering: C, vol. 93, p. 1073-1089, DOI:10.1016/j.msec.2018.08.049.

[3] Thanigaivelan, R., Arunachalam, R.M., Nithish, A., Venkatesh, S., Naveenkumar, P., Selvaganapathy, S., Siranjeevi, A.A.S. (2020). Optimization of laser and electrochemical process parameters for surface modification of hardness and hydrophobicity on 316L steel. Lasers in Engineering, vol. 45, no. 1-3, p. 69-84.

[4] Kovacı, H., Seçer, Y. (2020). Improved tribological performance of AISI 316L stainless steel by a combined surface treatment: Surface texturing by selective laser melting and plasma nitriding. Surface and Coatings Technology, vol. 400, art. ID 126178, DOI:10.1016/j.surfcoat.2020.126178.

[5] Liew, K.W., Kok, C.K., Ervina Efzan, M.N. (2016). Effect of EDM dimple geometry on friction reduction under boundary and mixed lubrication. Tribology International, vol. 101, p. 1-9, D0I:10.1016/j.triboint.2016.03.029.

[6] Yu, H., Deng, H., Huang, W., Wang, X. (2011) The effect of dimple shapes on friction of parallel surfaces. Proceedings of The Institution of Mechanical Engineers Part J: Journal of Engineering Tribology, vol. 225, no. 8, p. 693-703, DOl:10.1177/1350650111406045.

[7] Li, K., Hu, Y., Yao, Z. (2013). Experimental study of micro dimple fabrication based on laser shock processing. Optics \& Laser Technology, vol. 48, p. 216-225, D0l:10.1016/j. optlastec.2012.09.015.

[8] Dai, F.Z., Wen, D.P, Zhang, Y.K., Lu, J.Z, Ren, X.D., Zhou, J.Z. (2015). Micro-dimple array fabricated on surface of Ti6Al4V with a masked laser ablation method in air and water. Materials and Design, vol. 84, p. 178-184, D0I:10.1016/j. matdes.2015.06.137.

[9] Ahmed, A., Masjuki, H.H., Varman., M., Kalam, M.A., Habibullah, M., Al Mahmud, K.A.H. (2016). An overview of geometrical parameters of surface texturing for piston/ cylinder assembly and mechanical seals. Meccanica, vol. 51, p. 9-23, DOI:10.1007/s11012-015-0180-6.

[10] Won, S.J., Kim, H.S. (2019). Effects of laser parameters on morphological change and surface properties of aluminum alloy in masked laser surface texturing. Journal of
Manufacturing Processes, vol. 48, p. 260-269, D0I:10.1016/j. jmapro.2019.10.034.

[11] Puoza,J.C., Hua, X., Liu, Q., Kang, Z., Zhang, P. (2018). Manufacturing of micro-textures on metals by nanosecond laser micromachining. Advances in Materials and Processing Technologies, vol. 4, no. 1, p. 86-99, D0l:10.1080/237406 8X.2017.1406268.

[12] Behera, R.R., Babu, P.M., Gajrani, K.K., Sankar, M.R. (2017). Fabrication of micro-features on 304 stainless steel (SS-304) using Nd: YAG laser beam micro-machining. International Journal of Additive and Subtractive Materials Manufacturing, vol. 1, no. 3-4, p. 338-359, Dol:10.1504/ IJASMM.2017.089929.

[13] Lamraoui, A., Costil, S., Langlade, C., Coddet, C. (2010). Laser surface texturing (LST) treatment before thermal spraying: A new process to improve the substrate-coating adherence. Surface and Coatings Technology, vol. 205, p. S164-D167, DOI:10.1016/j.surfcoat.2010.07.044.

[14] Teleginski, V., Chagas, D.C., Costa de Oliveira, A.C., Santos, J.C.G., Azevedo, J.F., Riva, R., de Vasconcelos, G. (2014).Yb: fiber laser surface texturing of stainless steel substrate, with MCrAlY deposition and $\mathrm{CO}_{2}$ laser treatment. Surface and Coatings Technology, vol. 260, p. 251-259, D0l:10.1016/j. surfcoat.2014.06.076.

[15] Gajrani, K.K., Reddy, R.P.K., Sankar, M.R. (2016). Experimental comparative study of conventional, micro-textured and coated micro-textured tools during machining of hardened AISI 1040 alloy steel. International Journal of Machining and Machinability of Materials, vol. 18, no. 5-6, p. 522-539, DOI:10.1504/IJMMM.2016.078982.

[16] Sivaraj, D., Vijayalakshmi, K. (2019). Enhanced antibacterial and corrosion resistance properties of $\mathrm{Ag}$ substituted hydroxyapatite/functionalized multiwall carbon nanotube nanocomposite coating on 316 L stainless steel for biomedical application. Ultrasonics sonochemistry, vol. 59, art. ID 104730, DOI:10.1016/j.ultsonch.2019.104730.

[17] Thanigaivelan, R., Arunachalam, R.M. (2013). Optimization of process parameters on machining rate and overcut in electrochemical micromachining using grey relational analysis. Journal of Scientific and Industrial Research, vol. 72, no. 1, p.36-42.

[18] Pearson, K. (1901). On lines and planes of closest fit to systems of points in space. The London, Edinburgh, and Dublin Philosophical Magazine and Journal of Science, vol. 2, p. 559-572, DOl:10.1080/14786440109462720.

[19] Hotelling, H. (1993). Analysis of a complex of statistical variables into principal components. Journal of Educational Psychology, vol. 24, p. 417-441, DOl:10.1037/h0071325.

[20] Sonawane, S.A., Kulkarni, M.L. (2018). Optimization of machining parameters of WEDM for Nimonic-75 alloy using principal component analysis integrated with Taguchi method. Journal of King Saud University-Engineering Sciences, vol. 30, no. 3, p. 250-258, Dol:10.1016/j.jksues.2018.04.001.

[21] Wu, Z., Xing, Y., Huang, P., Liu, L. (2017). Tribological properties of dimple-textured titanium alloys under dry sliding contact. Surface and Coatings Technology, vol. 309, p. 21-28, DOl:10.1016/j.surfcoat.2016.11.045. 
[22] Thanigaivelan, R., Arunachalam, R.M., Jerald, J., Niranjan, T. (2011). Applications of Taguchi technique with fuzzy logic to optimise an electrochemical micromachining process. International Journal of Experimental Design and Process Optimisation, vol. 2, no. 4, p. 283-298, D0l:10.1504/ IJEDP0.2011.043565.

[23] Pradeep Kumar, G., Thanigaivelan, R., Arunachalam, R.M., Paramasivam, P. (2014). Experimental investigation of edm processing parameters on machining Al6063-12\% sic-5\% gr using response surface methodology. High Temperature Material Processes: An International Quarterly of HighTechnology Plasma Processes, vol. 18, no. 1-2, p. 27-43, DOI:10.1615/HighTempMatProc.2015014659.

[24] Zhao, W., Wang, W., Jiang, G., Li, B.Q., Mei, X. (2015). Ablation and morphological evolution of micro-holes in stainless steel with picosecond laser pulses. The International Journal of Advanced Manufacturing Technology, vol. 80, no. 9, p. 17131720, DOI:10.1007/s00170-015-7145-8. 